\section{Persistence of Gadolinium Contrast Enhancement in CSF: A Possible Harbinger of Gadolinium Neurotoxicity?}

Gadolinium chelates are relatively safe agents, with occasional hypersensitivity reactions and systemic toxicity with predominantly extracellular distribution and primarily glomerular clearance. Neurotoxicity has been estimated at approximately $1 \%$ on initial studies regarding the pharmacokinetics of gadolinium. ${ }^{1}$

We recently encountered a patient who, after receiving MR imaging and contrast MR angiography for a syncopal work-up, became encephalopathic. A follow-up MR imaging showed homogeneous T1 shortening and fluid-attenuated inversion recovery hyperintensity in the CSF (Fig 1). Lumbar puncture performed at that time yielded CSF that also demonstrated T1 shortening. Mass spectrometry yielded a concentration of $23,000 \mathrm{nmol} / \mathrm{mL}$ of gadolinium. The patient improved after serial hemodialysis sessions to baseline.

During interpretation of the second MR imaging, we recalled a similar report by Maramattom et al, ${ }^{2}$ who described a 57 -year-old woman with end stage renal failure, who developed encephalopathy after repeated intravenous gadolinium-enhanced MR imaging. On the basis of the temporal relationship of the gadolinium chelate administration and the resolution after dialysis, they suggested a diagnosis of gadolinium-related encephalopathy. ${ }^{2}$ The authors measured CSF gadolinium levels at $28,591 \mathrm{ng} / \mathrm{mL}(50 \mathrm{nmol} / \mathrm{mL})$. CSF gadolinium-related $\mathrm{T} 1$ shortening in the setting of renal failure was initially reported by Rai and Hogg, ${ }^{3}$ who emphasized the need for familiarity with this appearance, lest it be confused with other pathologic conditions.

In 2007, Arlt and Cepek ${ }^{4}$ described a more dramatic example of neurotoxicity in a 64-year-old woman with presumed spinal stenosis who inadvertently received $20 \mathrm{~mL}$ of intrathecal gadolinium dimeglumine $(10 \mathrm{mmol})$, which resulted in ataxia and delirium. They measured a CSF concentration of gadolinium of $23,365 \mathrm{nmol} / \mathrm{mL}$ on day 1 , which diminished to undetectable levels by day 5 .

In our patient, the paramagnetic effects in the patient's CSF also likely resulted from accumulation of gadolinium chelate administered during the initial MR imaging. Ultimately, the working diagnosis of gadolinium neurotoxicity was made because no other causes for the patient's encephalopathy were discovered. Although it is known that inorganic gadolinium salts are neurotoxic, it is unclear whether the unchelated gadolinium atoms, gadolinium chelates, or the chelating agents are the culprits for neurotoxicity.

Recent reports of neurotoxicity associated with gadolinium contrast should draw renewed attention to potential, though rare, adverse affects. When diffuse $\mathrm{T} 1$ shortening of the CSF is encountered, we suggest that this observation should prompt inquiry as to the patient's

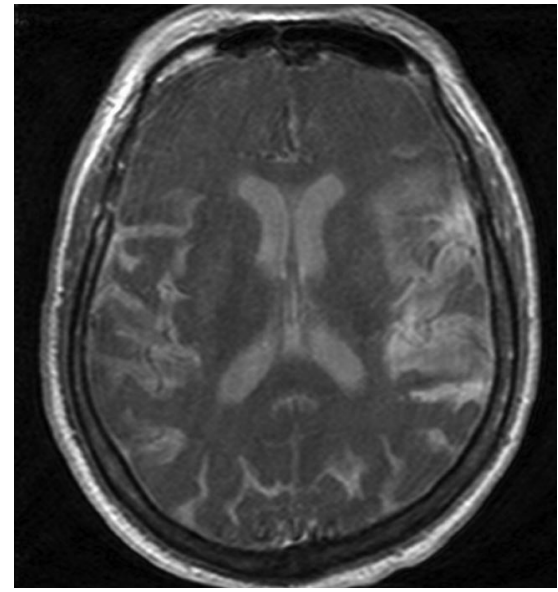

Fig 1. $T 1$ precontrast MR image shows homogenous $T 1$ shortening in the CSF.

renal status and discussion with the clinical team, who may be unaware of gadolinium-associated encephalopathy. Subclinical neurotoxicity may escape clinical attention if patients with renal failure are not specifically monitored, especially because gadolinium is efficiently removed by dialysis ( $95 \%$ at 3 sessions). ${ }^{1}$ We suggest that caution should be exercised when contemplating administering gadolinium chelates in this population due to diffusion of gadolinium chelates into the CSF and possible subsequent neurotoxicity.

\section{References}

1. Arsenault TM, King BF, Marsh JW Jr, et al. Systemic gadolinium toxicity in patients with renal insufficiency and renal failure: retrospective analysis of an initial experience. Mayo Clin Proc 1996;71:1150-54

2. Maramattom BV, Manno EM, Wijdicks EF, et al. Gadolinium encephalopathy in a patient with renal failure. Neurology 2005;64:1276-78

3. Rai AT, Hogg JP. Persistence of gadolinium in CSF: a diagnostic pitfall in patients with end-stage renal disease. AJNR Am J Neuroradiol 2001;22:1357-61

4. Arlt S, Cepek L, Rustenbeck HH, et al. Gadolinium encephalopathy due to accidental intrathecal administration of gadopentetate dimeglumine. $J \mathrm{Neu}$ rol 2007;254:810-12. Epub 2007 Apr 2

F.K. Hui

Department of Radiology

Division of Interventional Neuroradiology Emory MBNA Stroke Center

M. Mullins

Department of Radiology Division of Neuroradiology Emory University Hospital Atlanta, $\mathrm{Ga}$ 